\title{
THE EFFECT OF MHD ON FREE CONVECTION WITH PERIODIC TEMPERATURE AND CONCENTRATION IN THE PRESENCE OF THERMAL RADIATION AND CHEMICAL REACTION
}

\author{
B. ZIGTA and P.R. KOYA* \\ School of Mathematical and Statistical Sciences, Hawassa University \\ ETHIOPIA \\ E-mail: tzigta@yahoo.com; drkpraocecc@yahoo.co
}

\begin{abstract}
This paper studies the effect of magneto hydrodynamics on unsteady free convection between a pair of infinite vertical Couette plates. The temperature of the plates and concentration between the plates vary with time. Convection between the plates is considered in the presence of thermal radiation and chemical reaction. The solution is obtained using perturbation techniques. These techniques are used to transform nonlinear coupled partial differential equations to a system of ordinary differential equations. The resulting equations are solved analytically. The solution is expressed in terms of power series with some small parameter. The effect of various parameters, viz., velocity, temperature and concentration, has been discussed. Mat lab code simulation study is carried out to support the theoretical results. The result shows that as the thermal radiation parameter $R$ increases, the temperature decreases near the moving porous plate while it approaches to a zero in the region close to the boundary layer of the stationary plate. Moreover, as the modified Grashof number, i.e., based on concentration difference, increases, the velocity of the fluid flow increases hence the concentration decreases. An increase in both the chemical reaction parameter and Schmidt number results in decreased concentration.
\end{abstract}

Key words: MHD, unsteady free convection, thermal radiation, chemical reaction and Couette porous plates.

\section{Introduction}

Free convection arises in the fluid when temperature changes cause density variation leading to buoyancy forces acting on the fluid elements. Free convection occurs not only due to the temperature difference but also due to the concentration difference or combination of the two. The study of a free convection flow of an infinite vertical Couette plate has attracted many researchers over time due to its wide variety of applications in real world. The free convective flow has its applications in many areas including nuclear physics, ground water flow, coal gas, hydrology, soil physics, biological system, oil reservoir.

Magneto hydrodynamics or MHD is a field of study which relates electromagnetism with fluid mechanics. The main concept of MHD is that the magnetic field can induce currents in a moving conducting fluid, which in turn creates forces on the fluid and also changes the magnetic field itself. A fluid is said to be an electrically conducting fluid if it has the property of conducting electricity.

Maxwell's equations govern the electromagnetic property of fluids. The Navier - Stokes equation describes the momentum of a fluid. Since MHD has its applications in various areas, many scholars have been attracted to study the influence of the magnetic field in the boundary layer of an electrically conducting fluid. The application areas of magneto hydrodynamics include space dynamics, nuclear science, plasma physics and engineering design.

Some of the literature reviews related to the present study of magneto hydrodynamics convective flow of a fluid past a vertical plate are mentioned in what follows. A free convective steady flow past a vertical plate has been studied in [1 - 2]. The effects of viscous dissipation on the flow past an infinite

\footnotetext{
${ }^{*}$ To whom correspondence should be addressed
} 
vertical porous plate are studied. In this study, the amplitude of plate temperature oscillates is small. [3].The laminar free convection from a vertical plate is studied in [4].Free convection from a vertical plate through porous medium is studied [5].The nature of vertical free convection flow from the combined effects of thermal and concentration buoyancy is studied in [6].

In spite of the fact that the effects of thermal radiation and chemical reactions are very important and significant, they have not been considered in these studies. This omission encourages us to consider and study the effects of thermal radiation and chemical reactions. Thermal radiation plays an important role on both the flow and heat transfer process in fluids. Advanced energy conversion systems are operated at higher temperatures. In designing of these systems the effect of thermal radiation needs to be considered. Hot walls and the working fluid in these systems usually emit thermal radiation.

Thermal radiation has its applications in the areas of engineering, nuclear physics, missiles, aerodynamics. It occurs not only at high temperatures but also due to radiative heat transfer. An unsteady flow past a moving plate in the presence of free convection and radiation is investigated in [7].Free convective and concentration transfer of MHD through a porous medium with constant heat flux along an infinite vertical porous plate have been studied in [8]. The interaction between free convection and thermal radiation in a boundary layer flow past a vertical porous plate has been investigated in [9].The effects of thermal radiation on free convection in a porous medium of horizontal surfaces have been studied in [10].Free convection boundary layer flow with thermal radiation and mass transfer past a moving plate has been studied in [11].

The effects of thermal radiation on hydro magnetic fluid flows have been studied in [12].The effects of hydro magnetic flow past a vertical plate with and without as well as injection or suction have been studied in [13]. The hydro magnetic channel flow and temperature field are studied in [14].The MHD free convective flow of heat flux with an oscillating surface is studied in [15].The effect of oscillating temperature and concentration on an unsteady flow with constant suction is studied in [16]. An unsteady free convection oscillatory flow between parallel plates through a porous medium assuming periodic temperature variations has been investigated in [17].Recently, an oscillatory free convection flow past A plate how it is affected by magnetic field and thermal diffusion with viscous heating HAS BEEN studied in [18].

The effect of the magnetic field on the flow of a fluid between two vertical parallel Couette plates has been studied. The fluid considered here has the following properties, viz., viscous, incompressible, electrically conducting, Newtonian, thermal radiating and chemical reacting. Free stream velocity of the fluid is assumed to be fluctuating. Furthermore, we assume that the temperature and concentration of the fluid are also fluctuate with time [22].

The main objective of this paper is to study the effect of MHD on a free convection oscillatory Couette flow when the temperature and concentration oscillate with time in the presence of the thermal radiation and chemical reaction.

\section{Mathematical model and analysis}

Consider a two dimensional free convection Couette flow of an unsteady, viscous, incompressible, electrical conducting, Newtonian, thermal radiating and chemical reacting fluid bounded by two infinite vertical plates separated by a distance of $b$ as shown in Fig.1. The $\bar{x}$ axis is taken along the plate in the upward direction that is opposite to the direction of gravity and the $\bar{y}$ axis is taken normal to the vertical plate. The other stationary vertical plate is located at $\bar{y}=b$ where the temperature is given by the notation $\bar{T}_{b}$.

The vertical moving plate is located at $\bar{y}=0$ along the $\bar{x}$ axis where the temperature is $\bar{T}_{m}$ and the concentration is $\bar{C}_{m}$. The other stationary plate is located at $\bar{y}=b$ where the temperature is $\bar{T}_{b}$ and the concentration is $\bar{C}_{b}$. 
Initially, at $\bar{t}=0$, the stationary plate and the fluid are at the same temperature $\bar{T}_{b}$ and the concentration level of the fluid is $\bar{C}_{b}$ and is the same at all points. At any later time $\bar{t}>0$, the plate at $\bar{y}=0$ starts moving in its own plane with velocity $V$ and is heated by supplying heat at a constant rate while the stationary plate $\bar{y}=b$ is maintained at a constant temperature $\bar{T}_{b}$. It is assumed that the radiation heat flux in the $\bar{x}$ direction is negligible as compared to that of the $\bar{y}$ direction.

Furthermore, let us consider that the fluid is gray in color, has radiation absorbing nature, emits radiation, and is a non-scattering medium. The Roseland approximation is used to describe the radiative heat flux in the energy equation.

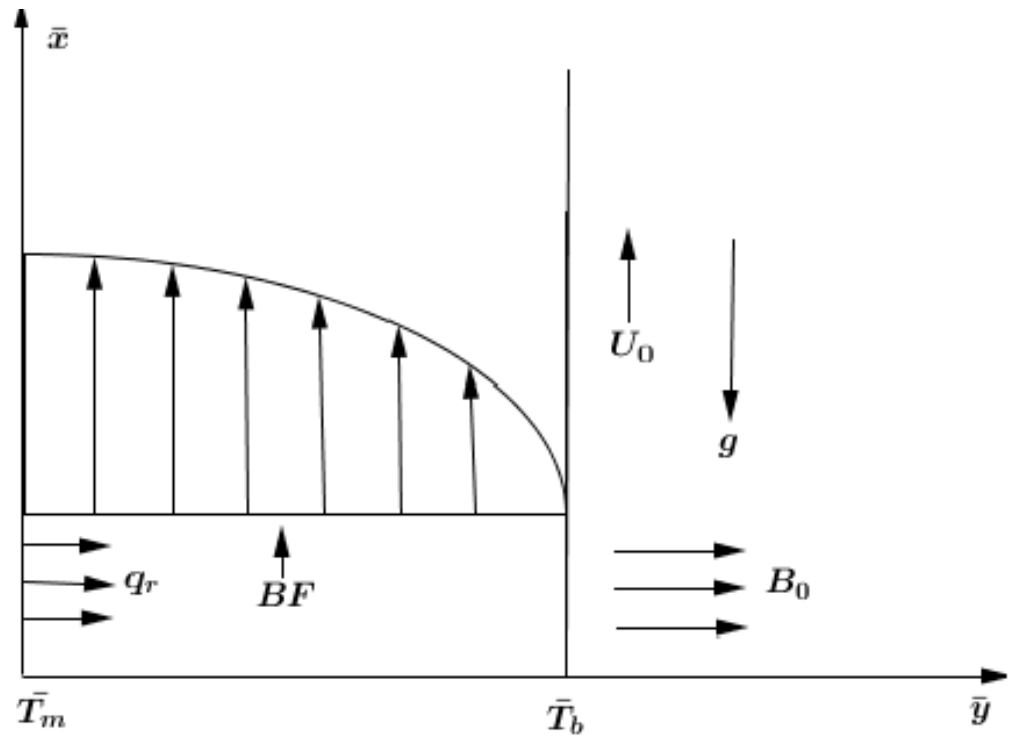

Fig.1. Flow configuration of the model problem.

We assume that the free stream velocity distribution has the form

$$
\bar{U}(\bar{t})=U_{o}\left(1+\varepsilon e^{i \overline{\bar{\omega}} \bar{t}}\right) .
$$

here $U_{o}$ represents the mean constant free stream velocity, $\bar{\omega}$ is the frequency and $\bar{t}$ is the time.

To derive the basic equations of the model the following assumptions are made:

1. The fluid properties such as temperature, viscosity, pressure, specific volume, specific gravity are constant.

2. All fluid properties except density in thermal and concentration buoyancy force term are constant.

3. The flow of the fluid is unsteady, laminar, incompressible, viscous, electrically conducting, Newtonian, thermal radiating and chemically reacting.

4. The magnetic field applied is transversal between the vertical plates.

5. The magnetic Reynolds numbers are small so that the induced magnetic field of the fluid is negligible.

6. The external electric field is supposed to be zero.

7. The electric field due to polarization of charges is negligible.

We now derive the governing equations of the present model based on the preceding assumptions:

The momentum equation of the model takes the form

$$
\frac{\partial \bar{u}}{\partial \bar{t}}=\frac{\partial \bar{U}}{\partial \bar{t}}+v \frac{\partial^{2} \bar{u}}{\partial \bar{y}^{2}}+g \beta\left(\bar{T}-\bar{T}_{b}\right)+g \beta_{c}\left(\bar{C}-\bar{C}_{b}\right)-\frac{J X B}{\rho}
$$


In Eq.(2.2), the vector cross product $J x B$ represents the Lorentz force. This term is a body force corresponding to the magneto hydrodynamic flow. The total magnetic field is represented by $B$. The density of the current is represented by $J$.

Using Ohm's law, the expression for the density of current $J$ can be constructed as

$$
J=\sigma(E+\bar{v} \times B)
$$

Here in Eq.(2.3), $\sigma$ is the electric conductivity, $E$ is the electric field and $\bar{v}$ is the velocity of the fluid. Further, the total magnetic field $B$ is expressed as $B=B_{o}+b_{1}$. Here $B_{o}$ is the imposed magnetic field and $b_{1}$ is the induced magnetic field. But, the model assumptions listed above imply that $E=0, b_{1}=0$ and $B=B_{0}$. Hence, the total magnetic field strengths can be expressed in component form as $\left(\begin{array}{lll}E, & B, & b_{1}\end{array}\right)=\left(\begin{array}{lll}0, & B_{0}, & 0\end{array}\right)$. Upon substituting $E=0$ in Eq.(2.3), it reduces to

$$
J=\sigma(\bar{v} \times B) .
$$

Also, the expression for the Lorentz force reduces and takes the form

$$
J x B=-\sigma B^{2} \bar{u} .
$$

In view of the result (2.5), Eq.(2.2) reduces to

$$
\frac{\partial \bar{u}}{\partial \bar{t}}=\frac{\partial \bar{U}}{\partial \bar{t}}+v \frac{\partial^{2} \bar{u}}{\partial \bar{y}^{2}}+g \beta\left(\bar{T}-\bar{T}_{b}\right)+g \beta_{c}\left(\bar{C}-\bar{C}_{b}\right)-\frac{\sigma B^{2}(\bar{u}-\bar{U})}{\rho} .
$$

The energy equation of the model can be expressed as

$$
\frac{\partial \bar{T}}{\partial \bar{t}}=\alpha \frac{\partial^{2} \bar{T}}{\partial \bar{y}^{2}}-\frac{1}{\rho C_{p}} \frac{\partial q_{r}}{\partial \bar{y}}
$$

The concentration equation of the model can be expressed as

$$
\frac{\partial \bar{C}}{\partial \bar{t}}=D \frac{\partial^{2} \bar{C}}{\partial \bar{y}^{2}}-k_{r}\left(\bar{C}-\bar{C}_{b}\right)
$$

Equations (2.6) - (2.8) govern the present model. Here, in Eqs (2.6)-(2.8), $\bar{u}$ and $\bar{v}$ are the velocity components along the positive directions of $\bar{x}$ and $\bar{y}$ coordinates respectively; $\alpha$ is the thermal diffusivity; $\rho$ is the density of the fluid; $g$ is the acceleration due to gravity; $\beta$ is the thermal expansion coefficient; $\beta_{c}$ is the concentration expansion coefficient; $\bar{T}$ is the temperature of the fluid in the boundary layer; $\bar{T}_{m}$ is the temperature of the moving plate; $\bar{T}_{b}$ is the temperature of the stationary plate; $q_{r}$ is the radiative heat flux; $D$ is the mass diffusivity; $\left[\left(1 / \rho C_{p}\right)\left(\partial q_{r} / \partial y\right)\right]$ is the thermal radiation effect; and $k_{r}\left(\bar{C}-\bar{C}_{b}\right)$ is the generative chemical reaction.

The physical interpretation of having the minus sign in Eqs (2.7) and (2.8) indicates that the mass diffusion represents 'generative chemical reaction'. Here in Eq.(2.8) chemical reaction is an exothermic reaction and in that heat is generated. However, the chemical reaction is said to be generative or exothermic 
if the chemical reaction parameter is negative, i.e., $k_{r}<0$. The minus sign of the radiative heat flux term $\left[\left(1 / \rho C_{p}\right)\left(\partial q_{r} / \partial y\right)\right]$ in Eq.(2.7) indicates emission of energy away from the objects.

Here, it is appropriate to point out that (i) the mass diffusion, (ii) the concentration species, and (iii) the concentration of the fluid. Hence, these terms are considered to be synonyms of each as far as our present work and presentation is concerned.

The boundary conditions for the model (2.6) - (2.8), can be chosen based on Fig.1, as

$$
\begin{aligned}
& \bar{y}=0, \quad \bar{u}=U_{0}\left(1+\varepsilon e^{i \overline{\bar{\omega}} \bar{t}}\right), \\
& \bar{T}=\bar{T}_{m}+\varepsilon\left(\bar{T}_{m}-\bar{T}_{b}\right) e^{i \overline{\bar{\omega}} \bar{t}}, \\
& \bar{C}=\bar{C}_{m}+\varepsilon\left(\bar{C}_{m}-\bar{C}_{b}\right) e^{i \overline{\bar{\omega}} \bar{t}}, \\
& \bar{y}=b, \quad \bar{u}=0, \quad \bar{T}=\bar{T}_{b}, \quad \bar{C}=\bar{C}_{b} .
\end{aligned}
$$

Using the Roseland approximation for the radiative heat transfer and the Roseland approximation for diffusion and also following the other works $[20-21]$, the expression for the radiative heat flux $q_{r}$ can be given as

$$
q_{r}=\left(\frac{-4 \sigma}{3 k_{s}}\right)\left(\frac{\partial \bar{T}^{4}}{\partial \bar{y}}\right)
$$

Here in Eq.(2.13), the parameters $\sigma$ and $k_{s}$ represent the Stefan Boltzmann constant and the Roseland mean absorption coefficient, respectively.

We now assume that the temperature differences within the fluid flow are sufficiently small so that $\bar{T}^{4}$ in Eq.(2.13) can be expressed as a linear function of $\bar{T}_{b}$ using the Taylor series expansion. The Taylor series expansion of $\bar{T}^{4}$ about $\bar{T}_{b}$, after neglecting the higher order terms, takes the form

$$
\bar{T}^{4} \cong 4 \bar{T}_{b}^{3} \bar{T}-3 \bar{T}_{b}^{4}
$$

Using Eqs (2.13) and (2.14) in Eq.(2.7), we obtain

$$
\frac{\partial \bar{T}}{\partial \bar{t}}=\alpha\left(\frac{\partial^{2} \bar{T}}{\partial \bar{y}^{2}}\right)+\left(\frac{1}{\rho C_{p}}\right)\left(\frac{16 \sigma \bar{T}_{b}^{3}}{3 k_{s}}\right)\left(\frac{\partial^{2} \bar{T}}{\partial \bar{y}^{2}}\right)+\left(\frac{Q_{0}}{\rho C_{p}}\right)\left(\bar{T}-\bar{T}_{b}\right) .
$$

In Eq.(2.15), the term $\left[\left(Q_{0} / \rho C_{p}\right)\left(\bar{T}-\bar{T}_{b}\right)\right]$ denotes heat absorption of the fluid.

\section{Non-dimensionalization of the model}

In order to solve the governing Eqs (2.6) - (2.8) of the model it is convenient and easy to deal with its dimensionless form. Hence, we find the dimensionless form of the model by introducing the following non- dimensional quantities 


$$
\begin{aligned}
& y=\bar{y} / b, \quad R=4 \sigma \bar{T}_{b}^{3} / \kappa k_{s}, \quad u=\bar{u} / U_{0}, \quad U=\bar{U} / U_{0}, \quad t=\bar{\varpi} \bar{t}, \quad \varpi=\bar{\varpi} b^{2} / \nu, \\
& \mathrm{Gc}=\left\{\left[g \beta_{c} b^{2}\left(\bar{C}_{m}-\bar{C}_{b}\right)\right] / \nu U_{0}\right\}, \quad \mathrm{Gr}=\left\{g \beta b^{2}\left(\bar{T}_{m}-\bar{T}_{b}\right) / \nu U_{0}\right\}, \quad \mathrm{Sc}=v / D, \quad \operatorname{Pr}=v / \alpha, \\
& M=\sqrt{\left(\sigma B^{2} b^{2} / \rho v\right)}, \quad C=\left[\left(\bar{C}-\bar{C}_{b}\right) /\left(\bar{C}_{m}-\bar{C}_{b}\right)\right], \quad \theta=\left[\left(\bar{T}-\bar{T}_{b}\right) /\left(\bar{T}_{m}-\bar{T}_{b}\right)\right] .
\end{aligned}
$$

After substituting the above non-dimensional quantities in Eqs (2.6) - (2.8) and after simple algebraic manipulations, the non-dimensional form of the model takes the following form

$$
\begin{aligned}
& \omega \frac{\partial u}{\partial t}=\omega \frac{\partial U}{\partial t}+\frac{\partial^{2} u}{\partial y^{2}}+\operatorname{Gr} \theta+\operatorname{Gc} C+M^{2}(u-U), \\
& \omega \frac{\partial \theta}{\partial t}=\frac{1}{p r}\left(1+\frac{4 R}{3}\right) \frac{\partial^{2} \theta}{\partial y^{2}}+\frac{Q_{o}}{\rho C_{p} v} \theta h^{2} \\
& \omega \operatorname{Sc} \frac{\partial C}{\partial t}=\frac{\partial^{2} C}{\partial y^{2}}-\frac{K_{r} C b^{2}}{D}
\end{aligned}
$$

The corresponding boundary conditions (2.9) - (2.12) take the form as

$$
\begin{aligned}
& y=0, \quad u=1+\varepsilon e^{i t}, \quad \theta=1+\varepsilon e^{i t}, \quad C=1+\varepsilon e^{i t}, \\
& y=1, \quad u=0, \quad \theta=0, \quad C=0 .
\end{aligned}
$$

The system of Eqs (3.1) - (3.3) together with the boundary conditions (3.4) - (3.5) constitutes the non-dimensional form of the present model.

\section{Analytical solution to the problem}

Now let us obtain an analytical solution to the non-dimensional form of the present model Eqs (3.1) (3.5). For that purpose let us assume that the amplitudes of the free stream velocity, temperature and concentration variation are very small positive quantities. Thus, the amplitude of the free stream velocity needs to be considered to be $0<\varepsilon \ll 1$.

Using perturbation techniques the solution of the model has the following form

$$
\begin{aligned}
& u(y, t)=u_{0}(y)+\varepsilon u_{l}(y) e^{i t}+o\left(\varepsilon^{2}\right), \\
& \theta(y, t)=\theta_{0}(y)+\varepsilon \theta_{l}(y) e^{i t}+o\left(\varepsilon^{2}\right), \\
& C(y, t)=C_{0}(y)+\varepsilon C_{l}(y) e^{i t}+o\left(\varepsilon^{2}\right) .
\end{aligned}
$$

Also, the free stream velocity takes the form 


$$
U=1+\varepsilon e^{i t}
$$

Substituting Eqs (4.1) - (4.4) in to Eqs (3.1) - (3.3), equating harmonic and non-harmonic terms and neglecting higher orders of $\varepsilon$ we obtain the following system of equations

$$
\begin{aligned}
& u_{o}{ }^{\prime \prime}-M^{2} u_{0}=-\mathrm{Gr} \theta_{0}-\mathrm{GcC}_{0}-M^{2}, \\
& u_{1}{ }^{\prime \prime}-\left(i \omega+M^{2}\right) u_{1}=-\mathrm{Gr} \theta_{1}-\mathrm{Gc}_{1}-\left(i \omega+M^{2}\right), \\
& \theta^{\prime \prime}{ }_{0}+\theta_{o}\left[\frac{Q_{o} h^{2} 3 \mathrm{Pr}}{\rho C_{p} v(3+4 R)}\right]=0, \\
& \theta^{\prime \prime}+\theta_{1}\left[\left(\frac{Q_{o} h^{2}}{\rho C_{p} v}-i \omega\right) \frac{3 \operatorname{Pr}}{(3+4 R)}\right]=0, \\
& C_{0}{ }^{\prime}-\frac{K_{r} C_{o} b^{2}}{D}=0, \\
& C_{1}{ }^{\prime \prime}-C_{l}\left(\frac{K_{r} b^{2}}{D}+i \omega \mathrm{Sc}\right)=0 .
\end{aligned}
$$

Further, the new boundary conditions corresponding to Eqs (3.4) - (3.5) are obtained as

$$
\begin{aligned}
& y=0, \quad u_{0}=1, \quad u_{1}=1, \quad \theta_{0}=1, \theta_{1}=1, \quad C_{0}=1, \quad C_{1}=1, \\
& y=1, \quad u_{0}=0, u_{1}=0, \theta_{0}=0, \theta_{1}=0, C_{0}=0, C_{1}=0 .
\end{aligned}
$$

Solving Eqs (4.5) - (4.10) together with the boundary conditions (4.11) - (4.12), we obtain the required analytical solutions as follows

$$
\begin{aligned}
& u_{0}(y)=A e^{M y}+B e^{-M y}+\frac{\mathrm{Gr}_{0}+\mathrm{Gc}_{0}}{M^{2}}+1, \\
& u_{1}(y)=A^{\prime} e^{p^{\prime} y}+B^{\prime} e^{-p^{\prime} y}+\frac{\mathrm{Gr}_{1}+\mathrm{GcC}_{1}}{i \omega+M^{2}}+1, \\
& \theta_{0}(y)=A_{1} e^{\xi y}+A_{2} e^{-\xi y}, \\
& \theta_{1}(y)=C_{1} e^{\beta x}+C_{2} e^{-\beta x} \\
& C_{0}(y)=d_{1} e^{\lambda_{1} y}+d_{2} e^{-\lambda_{1} y} \\
& C_{1}(y)=f_{1} e^{\lambda_{2} y}+f_{2} e^{-\lambda_{2} y}
\end{aligned}
$$


In Eqs (4.13) - (4.18), we have used the following notations

$$
\begin{aligned}
& A=\frac{M^{2}-(\mathrm{Gr}+\mathrm{Gc}) e^{-M}}{M^{2}\left(e^{-M}-e^{M}\right)}, \quad B=\frac{M^{2}-(\mathrm{Gr}+\mathrm{Gc}) e^{M}}{M^{2}\left(e^{M}-e^{-M}\right)}, \quad A^{*}=\frac{-(\mathrm{Gr}+\mathrm{Gc}) e^{2 p^{\prime}}}{i \omega+M^{2}}, \\
& B^{*}=\frac{(\mathrm{Gr}+\mathrm{Gc}) e^{2 p^{\prime}}}{\left(i \omega+M^{2}\right)\left(1-e^{2 p^{\prime}}\right)}, \quad P^{\prime}=\sqrt{i \omega+M^{2}}, \quad Q_{o}=\frac{16 \sigma \bar{T}_{b}^{2}}{k_{s}}, \\
& C_{1}=\frac{1}{e^{2 \beta}-1}, \quad C_{2}=\frac{e^{2 \beta}}{e^{2 \beta}-1}, \quad \lambda_{1}= \pm \sqrt{\frac{k_{r} b^{2}}{D}}, \quad \lambda_{2}= \pm \sqrt{\frac{k_{r} b^{2}}{D}+i \omega \mathrm{Sc}}, \\
& \xi=i \sqrt{\left(\frac{3 P r}{4 R+3}\right) \frac{Q_{o} h^{2}}{\rho C_{p} v}}, \quad \beta=i \sqrt{\left(\frac{3 \operatorname{Pr}}{4 R+3}\right)\left(\frac{Q_{o} h^{2}}{\rho C_{p} v}-i \omega\right)}, \quad A_{1}=\frac{-1}{e^{2 \xi}-1}, \quad A_{2}=\frac{e^{2 \xi}}{e^{2 \xi}-1}, \\
& d_{1}=\frac{1}{1-e^{2 \lambda_{1}}}, \quad d_{2}=\frac{e^{2 \lambda_{1}}}{e^{2 \lambda_{1}}-1}, \quad f_{1}=\frac{1}{1-e^{2 \lambda_{2}}}, \quad f_{2}=\frac{e^{2 \lambda_{2}}}{e^{2 \lambda_{2}}-1} .
\end{aligned}
$$

\section{Simulation study}

Consider here an unsteady magneto hydrodynamic free convection flow bounded between a pair of infinite vertical porous plates, of which one plate is moving, while the other remains stationary. The effects of different parameters such as the Grashof number based on temperature Gr, the modified Grashof number based on concentration difference Gc, Schmidt number Sc, the thermal radiation parameter $R$, the chemical reaction parameter $k_{r}$, Prandtl number Pr and Hartmann number M on the solution of the model have been considered for the simulation study.

For the sake of brevity or simplicity we have presented only the graphical representations of the temperature profile for different values of the parameter $R$, velocity profile for different values of $\operatorname{Pr}$ and also of the concentration profile for different values of the parameters $\mathrm{Gc}, \mathrm{Sc}$ and $k_{r}$.

Whenever the value of $\operatorname{Pr}$ is increased, the simulation study results in an increase in the viscosity and as a result a decrease in the velocity. However, whenever the value of Pr decreases, the simulation study results in a decrease in the viscosity and as a result an increase in the velocity and the same can be observed in the simulated graphs.

Similarly, whenever the value of $\operatorname{Pr}$ is increased, the simulation study results in a decrease of the thermal diffusivity and as a result a decrease of the thermal boundary layer. However, whenever the value of Pr decreases, the simulation study results in an increase in thermal diffusivity and the same can be observed in the simulated graphs.

If the value of the Hartmann number $M$ is increased the magnetic field of the fluid flow will also increase and as a result the velocity also decreases. Furthermore, the decrement in velocity results in decreasing the temperature. It can be put in simple words that whenever the Hartmann number increases, then the velocity as well as the thermal boundary layer will decrease. This result, being very straightforward, has not been verified in the simulation study.

In Fig.2, the simulated results of the influence of the Prandtl number Pr on the velocity are presented. The Prandtl number Pr is considered to be proportional to the viscosity $v$ but is inversely proportional to the thermal diffusivity $\alpha$. That is $\operatorname{Pr}=v / \alpha$. This theoretical fact has been verified 
successfully through the simulation study. Figure 2 shows that as Prandtl number Pr increases, the velocity of the fluid flow decreases.

In Fig.3, the simulated results of the influence of the thermal radiation parameter $R$ on the temperature are presented. It is a general and trivial fact that the parameter $R$ is inversely proportional to the temperature. That is, the increment in $R$ results in a decrement in the temperature. This fact has been shown in this figure. Furthermore, Fig. 3 shows that as the thermal radiation parameter $R$ increases, the temperature decreases near the moving porous plate while it approaches to a-zero in the region close to the boundary layer of the stationary plate.

Figure 4 illuistrates the influence of the modified Grashof number Gc on the concentration of the fluid. Naturally, the number Gc and the concentration are inversely proportional to each other. The same fact has been even shown by the present simulation study. Furthermore, Fig. 4 shows that as Gc increases the velocity of the fluid increases and hence, the concentration decreases.

In Fig.5, the influence of the Schmidt number Sc on the concentration of the fluid is presented. Naturally, the number Sc and the concentration are inversely proportional to each other. The same fact has been shown by the present simulation study. Furthermore, this figure shows that as the Sc increases the concentration of the fluid decreases. However, for different values of the Schmidt number Sc, the concentration profiles are plotted in Fig.5. Also, it is appropriate here to point out that physically Sc is related to both the concentration and hydrodynamic boundary layers.

In Fig.6, the influence of the chemical reaction parameter $K r$ on the concentration of the fluid is presented. Naturally, the number $K r$ and the concentration are inversely proportional to each other. The same fact has been even proved by the present simulation study. Furthermore, this figure shows that as $K r$ increases the concentration decreases. Also, in Fig. 6 it can be observed, as expected, that the increase in the values of the chemical reaction parameter $K r$ results in a decrease in concentration.

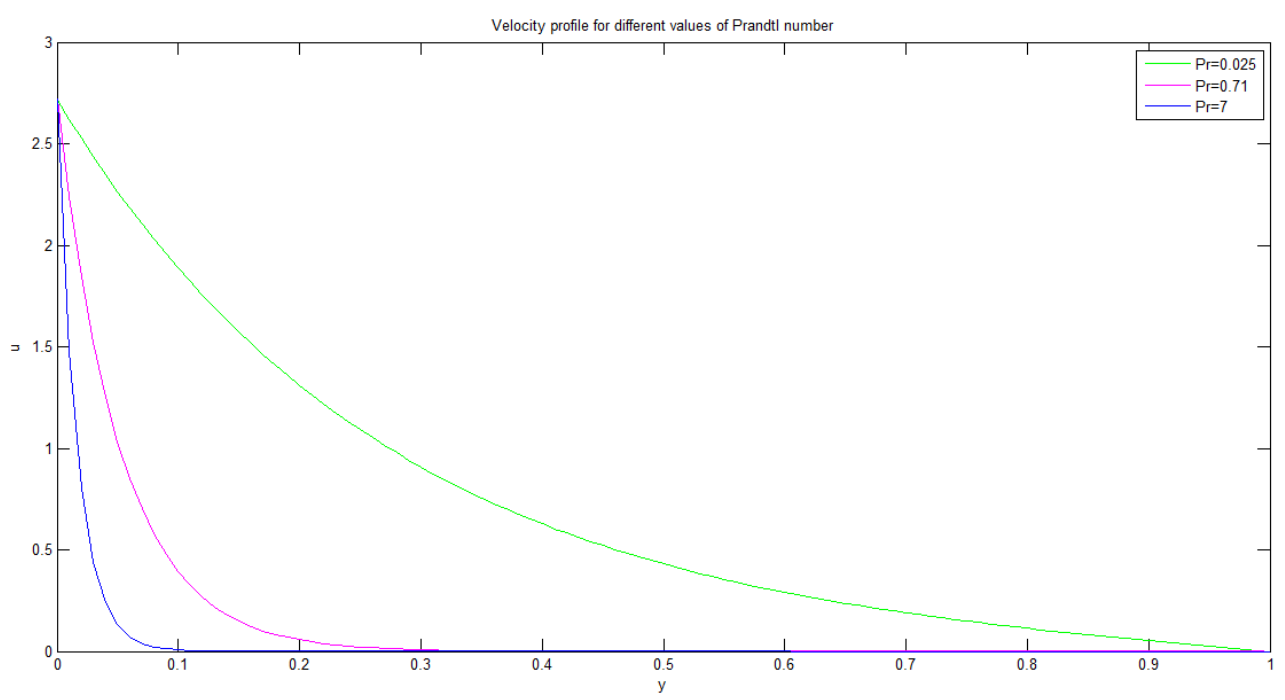

Fig.2. Velocity profile of the model for different values of the Prandtl number.

Figure 2 shows the influence of the Prandtl number Pr on the velocity of the fluid flow while holding the other parameters constant. The graph of the velocity is plotted by taking the distance from the moving plate to the stationary plate denoted by $y$ along the horizontal axis, while taking the value of the velocity denoted by $u$ along the vertical axis. The result shows that for a fixed value of the Prandtl number Pr the velocity $u$ starts from a constant value at the moving plate, and as $y$ increases the velocity decreases till it reaches a minimum value. 


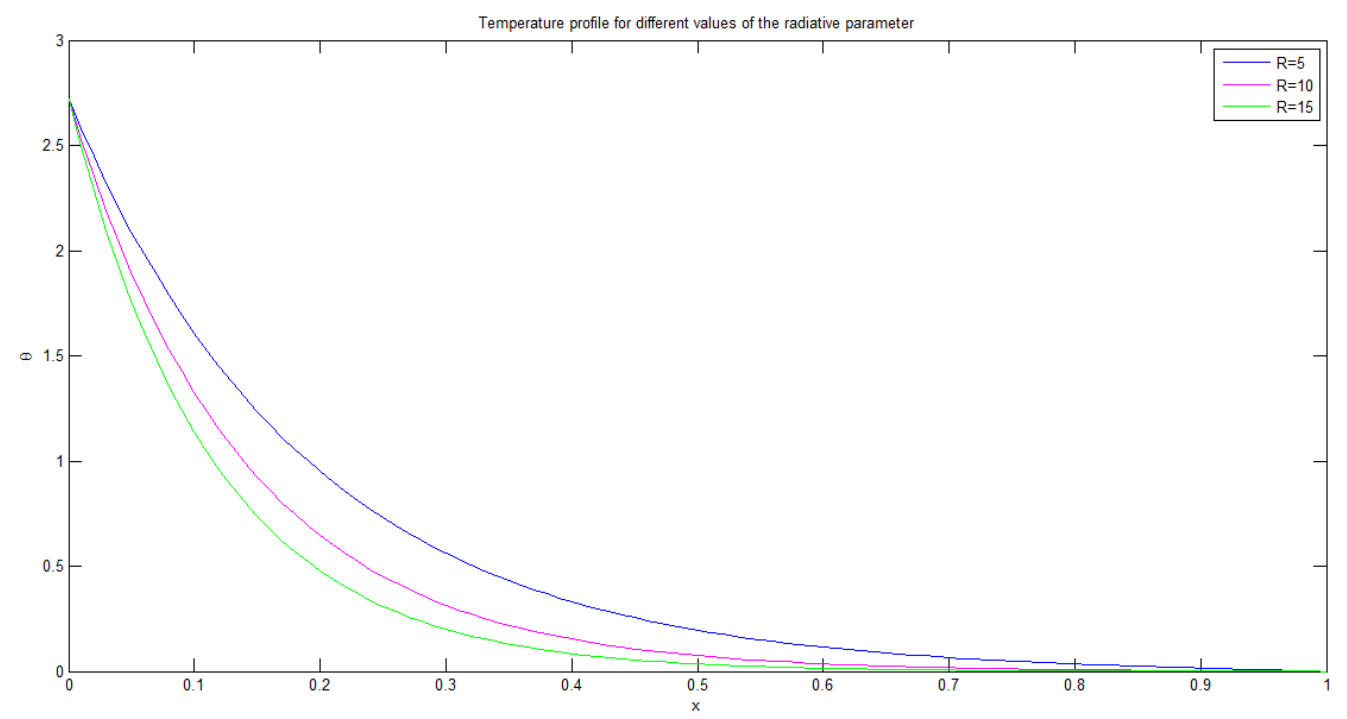

Fig.3. Temperature profile of the model for different values of the radiative parameter.

Figure 3 shows the influence of the thermal radiation parameter $R$ on the transfer of temperature while holding the other parameters constant. The graph of the temperature is plotted by taking the distance from the moving plate to the stationary plate denoted by $x$ along the horizontal axis, while taking the value of temperature denoted by $\theta$ along the vertical axis.

The result shows that for a fixed value of the thermal radiation parameter $R$, the temperature $\theta$ starts from a constant value at the moving plate, and as $x$ increases the temperature decreases till it reaches a minimum value. Nevertheless, the temperature at the moving plate is always at a higher value than that at the stationary plate.

As the temperature of the moving plate is higher than that of the stationary plate, the temperature is expected to transfer from the moving plate towards the stationary plate. This fact has been proved in this simulation.

It can also be observed from the simulation that: (i) the value of temperature does not change with the change in $R$ at the moving plate (ii) the minimum value of temperature, occurs between the two plates, it decreases with the increase of $R$, i.e., the minimum value of temperature is inversely proportional to that of $R$.

Figure 4 shows the influence of the modified Grashof number Gc on the concentration profile of the fluid while holding the other parameters constant. The graph of the concentration value is plotted by taking the distance from the moving plate to the stationary plate denoted by $y$ along the horizontal axis, while the value of fluid concentration denoted by $\eta$ is taken along the vertical axis.

The result shows that for a fixed value of the modified Grashof number Gc, the value of fluid concentration denoted by $\eta$ starts from a maximum constant value at the moving plate, and as $y$ increases the concentration $\eta$ decreases and reaches a minimum constant value at the stationary plate. Nevertheless, the measure of fluid concentration at the moving plate is always at a higher value than that at the stationary plate.

As the concentration of the fluid at the moving plate is higher than that at the stationary plate, the concentration is expected to move from the moving plate towards the stationary plate. Fluids diffuse from the locations of higher concentrations to those of lower concentrations. This fact has been shown in this simulation.

Also, it can be observed from-the simulation that: (i) the value of concentration of the fluid does not change with the change in Gc at the moving plate (ii) the value of concentration of the fluid, occurring between the two plates, decreases with the increase of Gc, i.e., the value of concentration of the fluid is 
inversely proportional to that of Gc (iii) the value of concentration of the fluid goes to zero at the stationary plate irrespective of the value of Gc.

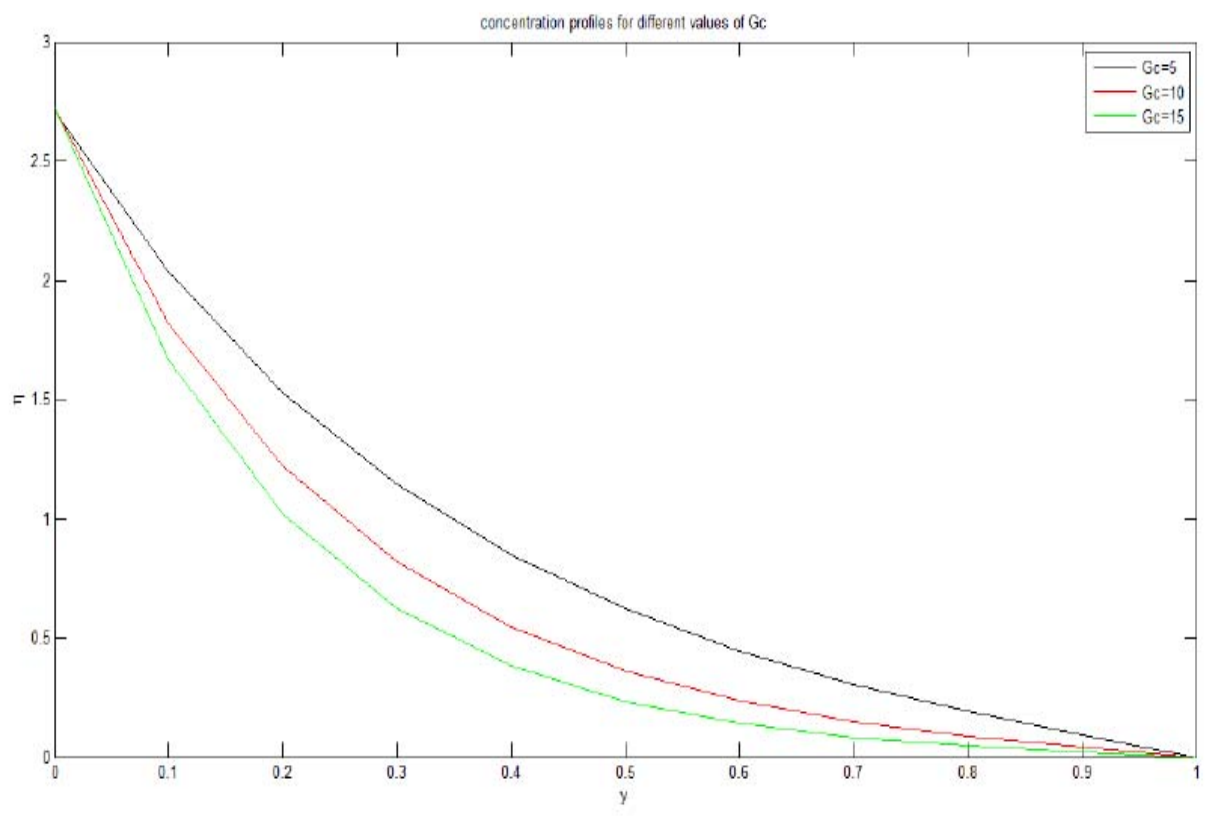

Fig.4. Concentration profile of the model for different values of the modified Grashof number Gc.

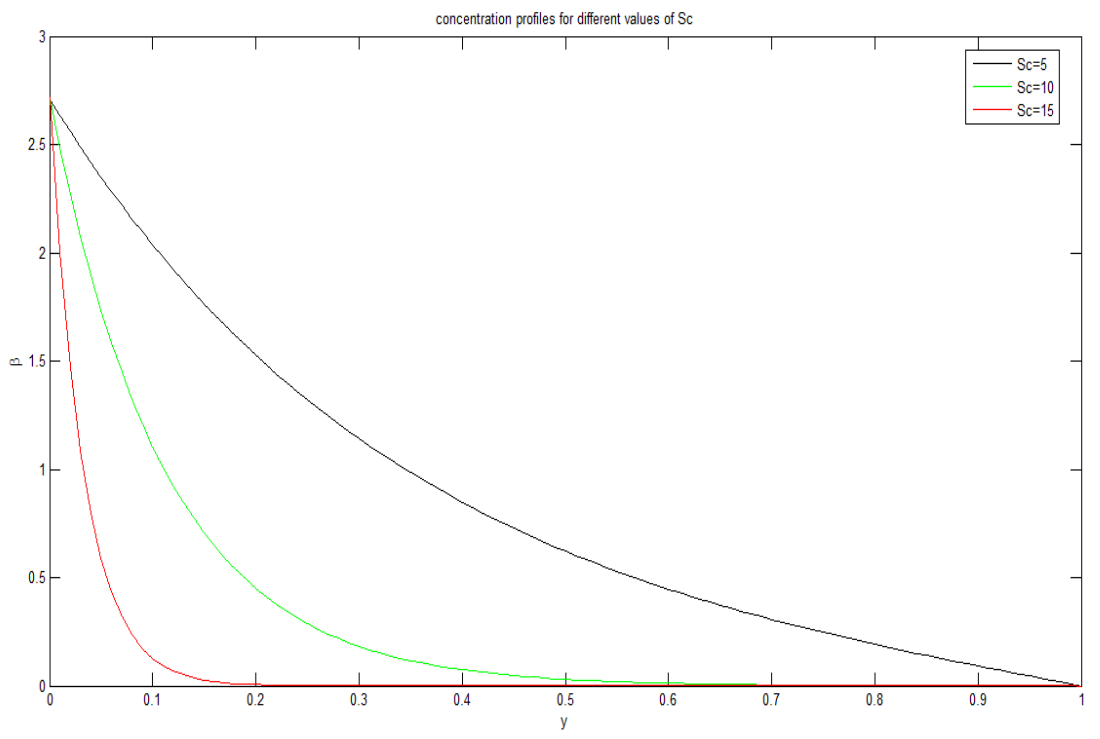

Fig.5. Concentration profile of the model for different values of the Schmidt number Sc.

Figure 5 shows the influence of the Schmidt number Sc on the concentration profile of the fluid while holding the other parameters constant. The graph of the concentration value is plotted by taking the distance from the moving plate to the stationary plate denoted by $y$ along the horizontal axis, while the value of fluid concentration denoted by $\beta$ is taken along the vertical axis.

The result shows that for a fixed value of the Schmidt number Sc, the value of fluid concentration denoted by $\beta$ starts from a maximum constant value at the moving plate, and as $y$ increases the 
concentration $\beta$ decreases and reaches a zero constant value at the stationary plate. Nevertheless, the measure of fluid concentration at the moving plate is always at a higher value than that at the stationary plate.

As the concentration of the fluid at the moving plate is higher than that at the stationary plate, it is expected to move from the moving plate towards the stationary plate. Fluids diffuse from the locations of higher concentrations to those of lower concentrations. This fact has been proved in this simulation.

It can also be observed from the simulation that: (i) the value of concentration of the fluid does not change with the change in Sc at the moving plate (ii) the value of concentration of the fluid, occurring between the two plates, decreases with the increase of Sc, i.e., the value of concentration of the fluid is inversely proportional to that of Sc (iii) the value of concentration of the fluid goes to zero at the stationary plate irrespective of the value of Sc.

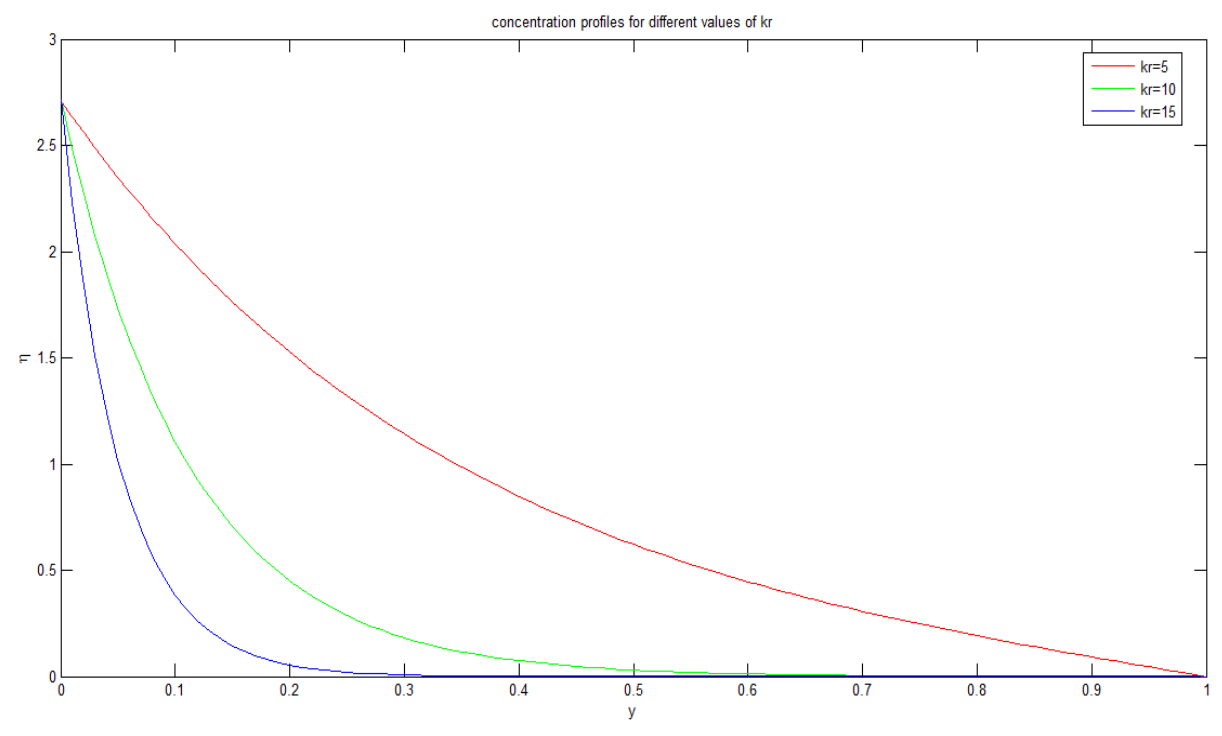

Fig.6. Concentration profile of the model for different values of the chemical reaction parameter $\mathrm{Kr}$.

Figure 6 shows the influence of the chemical reaction parameter $K r$ on the concentration profile of the fluid while holding the other parameters constant. The graph of the concentration value is plotted by taking the distance from the moving plate to the stationary plate denoted by $y$ along the horizontal axis, while the value of the fluid concentration denoted by $\eta$ is taken along the vertical axis.

The result shows that for a fixed value of the chemical reaction parameter $K r$, the value of the fluid concentration denoted by $\eta$ starts from a maximum constant value at the moving plate, and as $y$ increases the concentration $\eta$ decreases and reaches a zero constant value at the stationary plate. Nevertheless, the measure of fluid concentration at the moving plate is always at a higher value than that at the stationary plate.

As the concentration of the fluid at the moving plate is higher than that at the stationary plate, the concentration is expected to move from the moving plate towards the stationary plate. Fluids diffuse from the locations of higher concentrations to those of lower concentrations. This fact has been shown in this simulation.

Also, it can be observed from that simulation that: (i) the value of concentration of the fluid does not change with the change in $K r$ at the moving plate (ii) the value of concentration of the fluid, occurring between the two plates, decreases with the increase of $K r$, i.e., the value of concentration of the fluid is inversely proportional to that of $K r$ (iii) the value of concentration of the fluid goes to zero at the stationary plate irrespective of the value of $K r$. 


\section{Conclusions}

In this paper, the effects of thermal radiation and chemical reaction on an unsteady magneto hydrodynamics free convection between a pair of infinite vertical porous plates of which one is moving while the other is stationary, are considered.

The configuration of the fluid flow field is affected by the vertical motion of the hot plate. However, the vertical motion of the hot plate is due to the radiation, buoyancy force and shear induced force. The effects of various parameters $\mathrm{Gc}, \mathrm{R}, \mathrm{Sc}$ and $\mathrm{k}_{\mathrm{r}}$ on the velocity, temperature and concentration of the fluid have been discussed.

Perturbation technique is applied to convert the governing non-linear partial differential equations into a system of ordinary differential equations which have been solved analytically.

Furthermore, the effects of the Prandtl number and Hartmann number on the fluid flow have been discussed. Increasing the Prandtl number results in decreasing the thermal boundary layer but in case of a smaller Prandtl number the boundary layer is thicker. From this observation, it can be concluded that the thickness of the boundary layer has a direct relationship with viscosity. That is, as viscosity increases the thickness of the boundary layer increases and vice versa. This is also true in the case of the concentration boundary layer.

\section{Nomenclature}

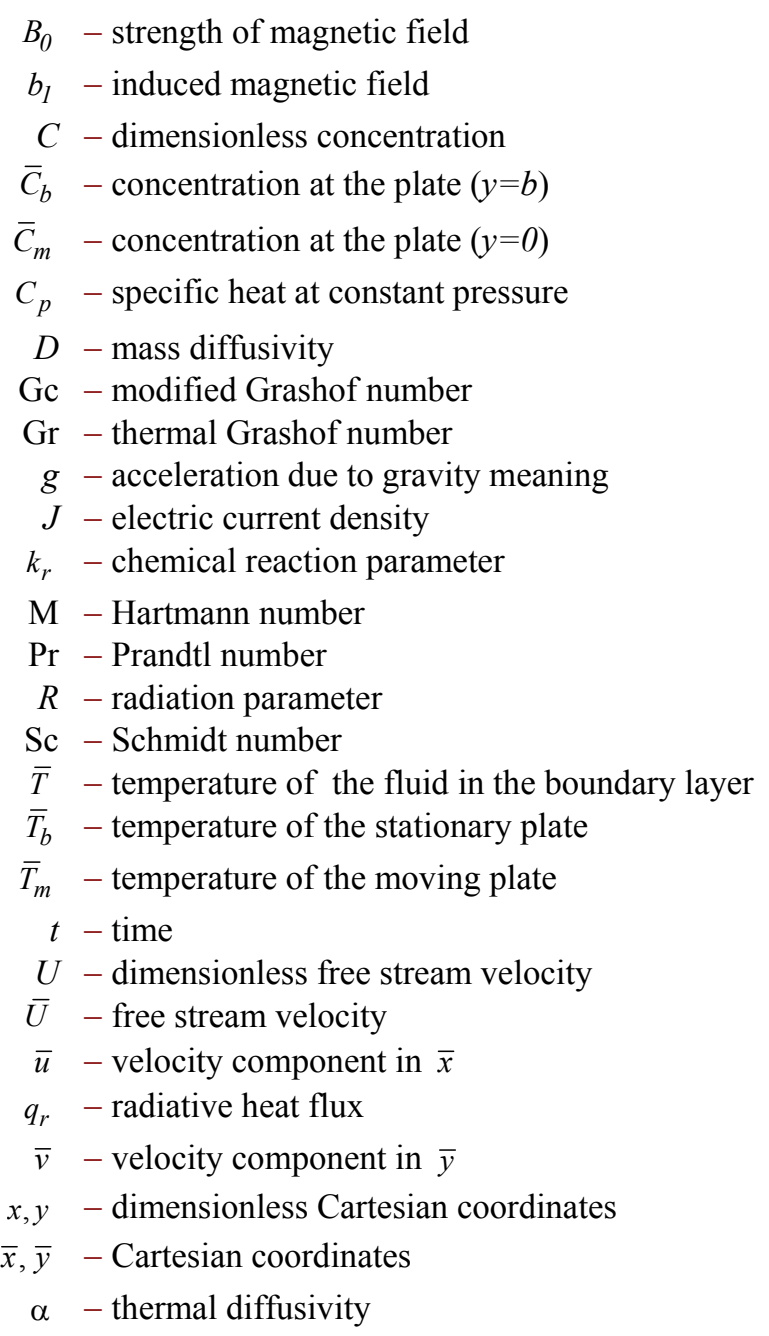


$\beta-$ thermal expansion coefficient

$\beta_{c}-$ concentration expansion coefficient

$\varepsilon \quad$ - amplitude of free stream velocity

$\theta$ - dimensionless temperature

$\kappa-$ thermal conductivity

$\mu-$ dynamic viscosity

$v-$ kinematic viscosity

$\sigma-$ electric conductivity

$\omega-$ frequency of oscillation

\section{References}

[1] Ostrich S. (1952): Laminar natural convection flow and heat transfer of fluids with and without hear sources in channels with constant wall temperature. - NACA TN, pp.2863.

[2] Ibid (1953): New aspects of natural convection heat transfer. Trans. Am. Soc. Mec. Engrs., vol.75, pp.1287-1290.

[3] Soundalgekar V.M. (1972): Viscous dissipation effects on unsteady free convective flow past an infinite vertical porous plate with constant suction. Int. J. Heat Mass Transfer, vol.15, pp.1253-1261.

[4] Martynenko O.G., Berezovsky A.A. and Sokovishin Yu.A. (1984): Laminar free convection from a vertical plate. - Int. J. Heat Mass Transfer, vol.27, pp.869-881.

[5] Harris S.D., Ingham D.B. and Pop I. (1997): Free convection from a vertical plate in a porous medium subjected to a sudden change in surface temperature. - Int. Comm. Heat Mass Transfer, vol.24, pp.543-552.

[6] Gebhart B. and Pera L. (1971): The nature of vertical natural convection flow from the combined buoyancy effects on thermal and mass diffusion. - Int. J. Heat Mass Transfer, vol.14, pp.2024-2050.

[7] Mansour M.H. (1990): Radiative and free convection effects on the oscillatory flow past a vertical plate. Astrophysics and Space Science, vol.166, pp.26-75.

[8] Raptis A. and Kafoussias N.G. (1982): MHD free convection flow and mass transfer through porous medium bounded by an infinite vertical porous plate with constant heat flux. - Canadian J. Physics, vol.60, pp.17251729.

[9] Abdus Sattar M.D. and Hamid Kalim M.D. (1996): Unsteady free-convection interaction with thermal radiation in a boundary layer flow past a vertical porous plate. J. Mathematics and Physical Sciences, vol.30, pp.25-37.

[10] Bakier A.Y. and Gorla R.S.R. (1996): Thermal radiation effects on horizontal surfaces in saturated porous medium. - Transport Porous Media, vol.23, pp.357-61.

[11] Makinde O.D. (2005): Free convection flow with thermal radiation and mass transfer past a moving vertical porous plate. - Int. Communications on Heat and Mass Transfer, vol.32, pp.1411-1419.

[12] Abdelkhalek M.M. (2007): Thermal radiation effects on hydromagnetic flow. - Computer Assisted Mechanics and Engineering Sciences, vol.14, pp.471-484.

[13] Greenspan H.P. and Carrier G.F. (1959): the MHD flow past a flat plate. - J. Fluid Mech., vol.6, pp.77-96.

[14] Attia H.A. and Kotab N.A. (1996): MHD flow between two parallel plates with heat transfer. - Acta Mech., vol.117, pp.215-220.

[15] Hossain M.A., Das S.K. and Pop I. (1998): Heat transfer response of MHD free convection flow along a vertical plate to surface temperature oscillation. - Int. J. Non-Linear Mech., vol.33, pp.541-53.

[16] Sharma B.K., Sharma P.K. and Chaudhary R.C. (2009): Effect of fluctuating surface temperature and concentration on unsteady convection flow past an infinite vertical plate with constant suction. - Heat Transfer Research, vol.40, No.6, pp.1-15. 
[17] Sharma P.K., Sharma B.K. and Chaudhary R.C. (2007): Unsteady free convection oscillatory Couette flow through a porous Medium with periodic wall temperature. - Tamkang Journal of Mathematics, vol.38, pp.93102.

[18] Sharma P.K. and Singh Ch. (2013): Effect of MHD and thermal diffusion on natural convection oscillatory flow past plate with viscous heating. - Math. Sci. Lett., vol.2, No.2, pp.79-86.

[19] Hossain M.A., Alim M.A. and Rees D.A.S. (1999): The effect of radiation on free convection from a porous vertical plate. - Int. J. Heat Mass Transfer, vol.42, pp.181-91.

[20] Raptis A. (1998): Flow of micro polar fluid past a continuously moving plate by the presence of radiation. - Int. J. Heat Mass Transfer, vol.41, pp.2865-2866.

[21] Brewster M.A (1992): Thermal Radiative Transfer and Properties. - New York: John Wiley and Sons.

[22] Sharma P.K. and Dutt M. (2014.): MHD oscillatory free convection flow past parallel plates with periodic temperature and concentration. - Universal Journal of Applied Mathematics, vol.2, No.7, pp.264-75, Doi: 10.13189/ujam.2014,020702.

Received: January 6, 2017

Revised: October 12, 2017 